\title{
11 DBS on Natural Data Sets
}

Several real-world data sets are used in this chapter to show that Databionic swarm (DBS) is able to find clusters in a variety of cases. The leukemia data set is based on luminance measurements of 7747 different active or non-active genes in 554 human subjects. The World GDP data set is a multivariate time series that consists of monetary values for 190 countries from 1970 to 2010 . The Tetragonula data set contains 13 string variables consisting of pairs of alleles for 13 microsatellite loci in bees. In each case, suitable preprocessing and a correctly chosen distance definition make it possible for DBS to cluster and visualize the data such that the known knowledge is reproduced.

\subsection{Types of Leukemia}

The leukemia data set consists of 7747 variables for 554 subjects (for details, see chapter 3). Of the subjects, 109 were healthy, 15 were diagnosed with acute promyelocytic leukemia (APL), 266 had chronic lymphocytic leukemia (CLL), and 164 had acute myeloid leukemia (AML). The leukemia data set is a high-dimensional data set with natural clusters specified by the illness status and defined by discontinuities (for details, see chapters 3 and 9).

Figure 11.1 shows a visualization of the healthy patients and the patients diagnosed with these three major types of leukemia. The four groups are well separated by mountains, with the subjects represented by points of different colors. Magenta points indicate healthy subjects, whereas points of other colors indicate ill subjects. The automatic clustering of DBS is able to separate the four groups with an accuracy of 99.6\%. Two outliers can be seen in Figure 11.1, marked with red arrows. These green and yellow outliers cannot be explained without deanonymization of the patients, which was not feasible for the author. They may be misclassified, but a future publication will address this diagnostic problem ${ }^{72}$.

\subsection{World Gross Domestic Product (World GDP)}

The World GDP data set, published in [Leister, 2016], consists of data on the gross domestic product (GDP) per capita for 160 countries over the past 40 years (see chapter 9 for details). The dynamic time warping (DTW) distances were calculated using the R package dtw [Giorgino, 2009], which computes the optimal alignment between two time series [Giorgino, 2009]. The homogeneity of the cluster structures of DBS is visualized in a silhouette plot in Figure 11.4, the result of the DBS method in Figure 11.2 shows this clear cluster structure and it is confirmed by the heatmap in Figure 11.3.

As the rules deduced through Classification and Regression Tree (CART) analysis show in Figure 11.5, the clusters are defined by a tragic event that occurred in 2001, the crashing of airplanes into the World Trade Center. In its aftermath, "the world economy was experiencing its first synchronized global recession in a quarter-century” [Makinen, 2002, p. 17].

\footnotetext{
${ }^{72}$ It should be remarked that a data-driven DBS clustering does not reproduce the classification(s) of AML (like FAB subtypes) or CLL of research in this area, e.g. [Bene et al., 1995; Bennett et al., 1985; Vardiman et al., 2009; Haferlach et al., 2010], for CLL see [Rosenwald et al., 2001]. See also p. 30 fn. 19.
} 


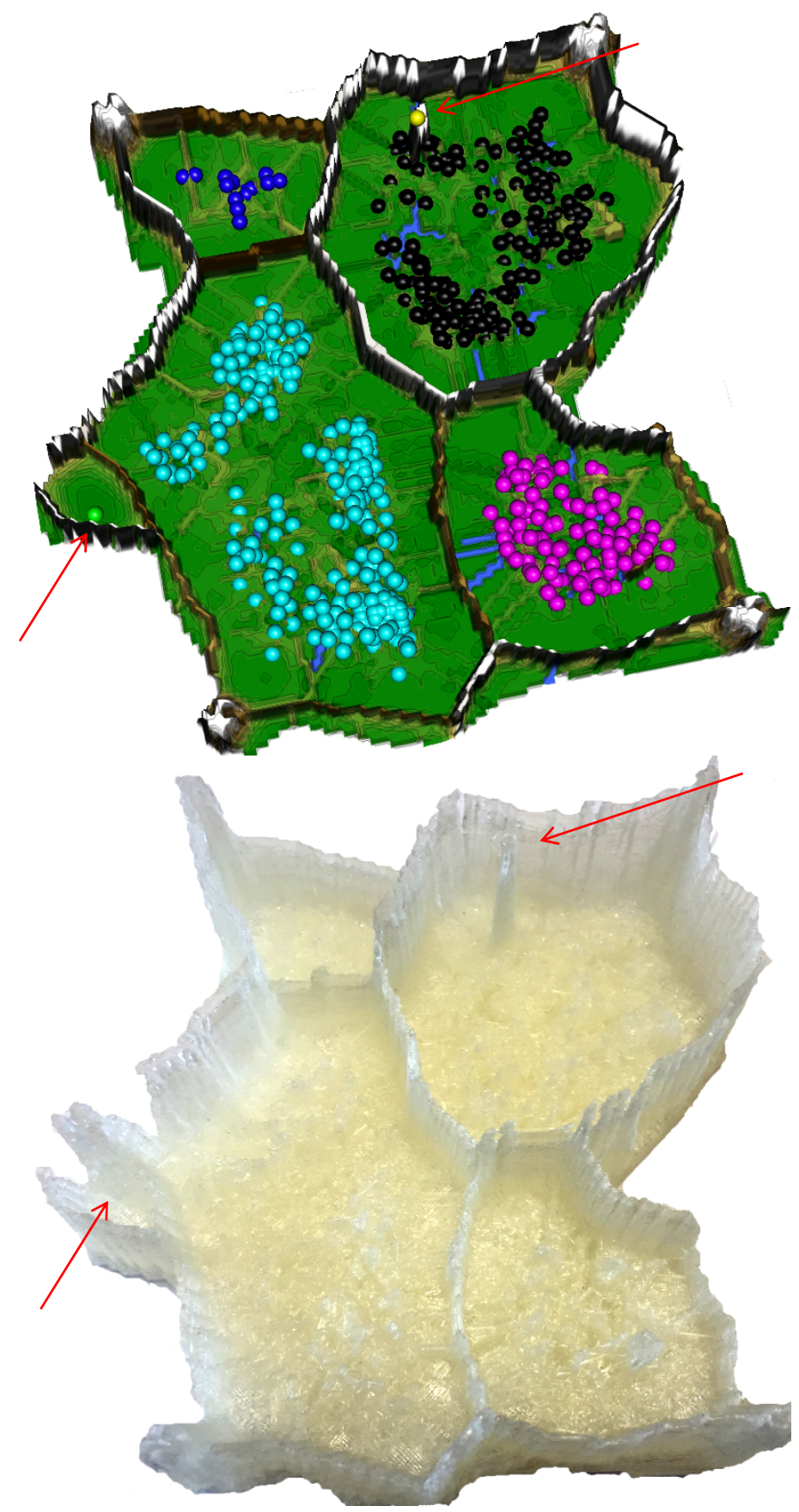

Figure 11.1: Topographic map with DBS clustering results for the leukemia data set, showing six clusters and an accuracy of $99.6 \%$ in comparison with the prior classification of four leukemia statuses.

Top: healthy (magenta), AML (cyan), APL (blue), and CLL (black). Two outliers are marked with red arrows: an APL outlier (green) and a CLL outlier (yellow).

Bottom: 3D print (see [Thrun et al., 2016a]), colors are not available yet due to technical limitations. 
Therefore, the first cluster consists mostly of African and Asian countries, which were generally unaffected by this event, and the second cluster consists of American and European countries, which were affected. The outlier is Equatorial Guinea, where the first Parliamentary elections since 1968 were held in 1983. Equatorial Guinea shows the smallest variance in its GDP, which is mostly based on oil - this small country, with an area of 28,000 square kilometers, is one of sub-Saharan Africa's largest oil producers.

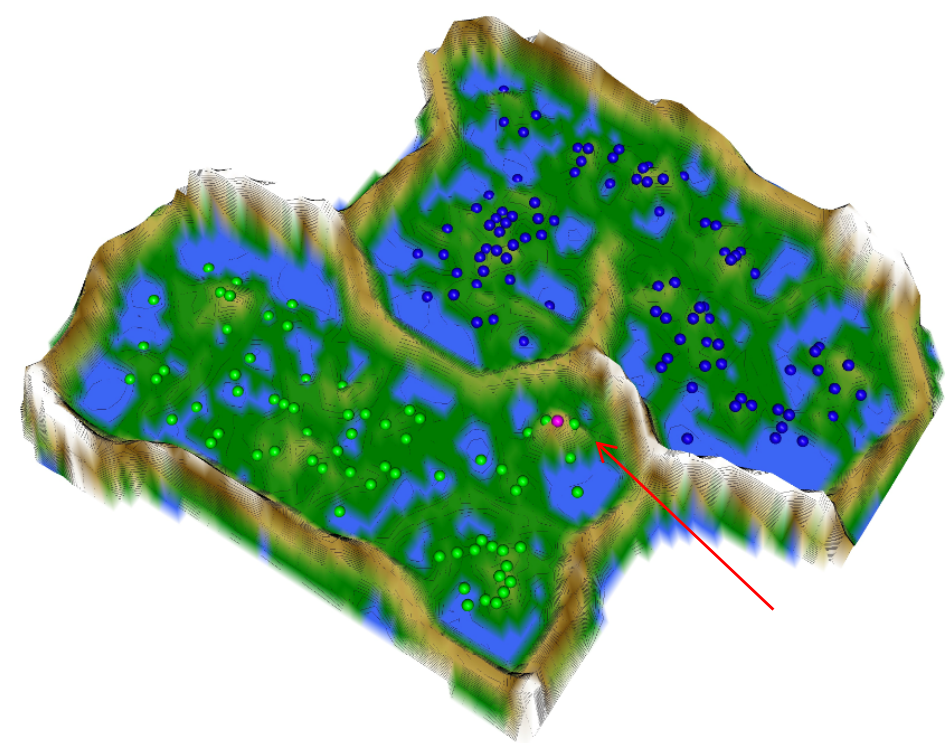

Figure 11.2: Topographic map of the DBS clustering of the World GDP data set shows two distinctive clusters. There is one outlier, colored in magenta and marked with a red arrow.

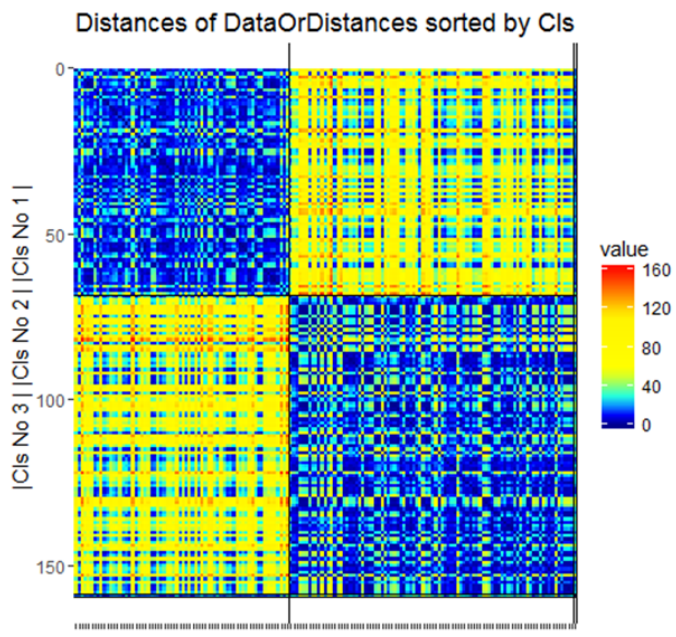

|Cls No 1 | |Cls No 2 ||Cls No $3 \mid$

Figure 11.3: Heatmap of the dynamic time warping (DTW) distances for the World GDP data set shows a small variance of intracluster distance. 


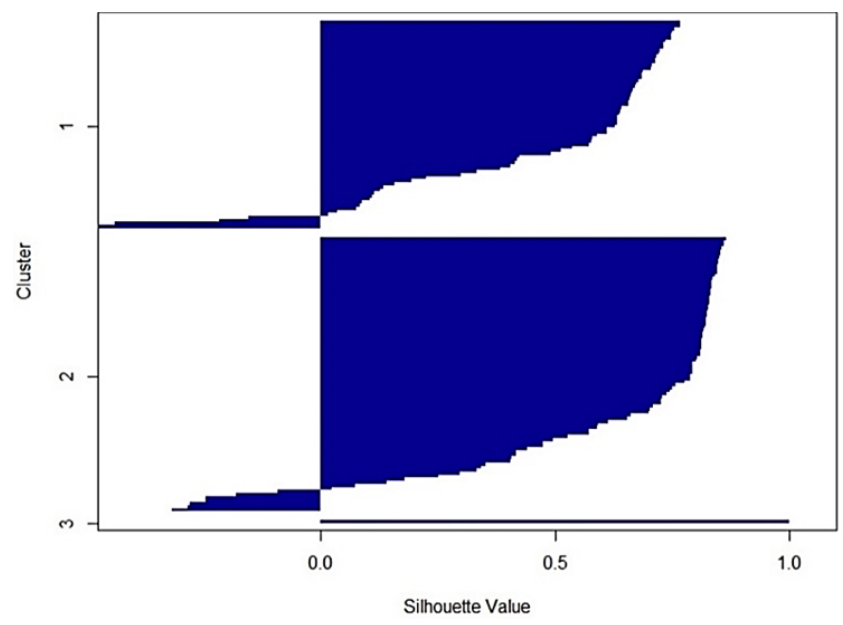

Figure 11.4: Silhouette plot of the DBS clustering results for the World GDP data set indicates that data points (y-axis) above a value of 0.5 (x-axis) have been assigned to an appropriate cluster.

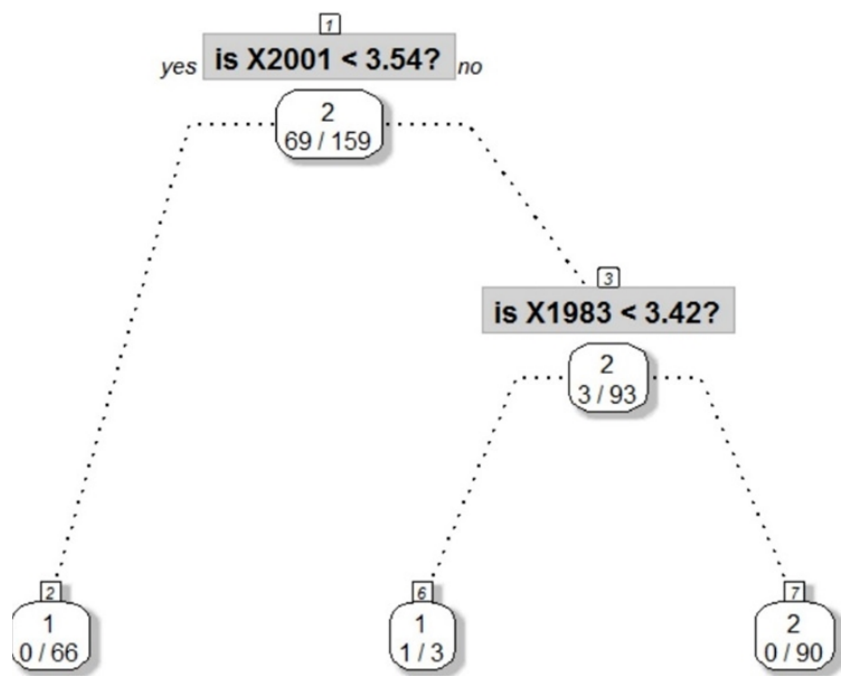

Figure 11.5: Classification and Regression Tree (CART) analysis rules for the clusters. The two main clusters are defined only by an event in 2001 .

\subsection{Tetragonula Bees}

The Tetragonula data set was published in [Franck et al., 2004] and contains the genetic data of 236 Tetragonula bees from Australia and Southeast Asia, expressed using 13 variables (for details, see chapter 9), with a specific distance definition.

The shared allele distance is described in [Hausdorf/Hennig, 2010, p. 493] as follows:

"[The distance is] defined as one minus the proportion of alleles shared by 2 individuals averaged over loci. Loci with missing values are not considered in the pairwise distance calculation. In the presence of missing values, this distance measure is not necessarily a metric." 
For the distance calculation, the R package fpc of [Hausdorf/Hennig, 2010] was used with the distance introduced by [Bowcock et al., 1994].

The first DBS visualization implied the existence of 8 clusters and two pairs of outliers. Hence, 100 trials of Pswarm projection and DBS clustering with $\mathrm{k}=10$ clusters were generated, and the best one (i.e., the one with the smallest Delaunay clustering error (DCE)) was chosen (Figure 11.7). The silhouette plot indicates a hyperspherical cluster structure (Figure 11.6) and the heatmap of the distances in Figure 11.9 confirmed the DBS clustering. This application of DBS illustrated the possibility of using multiple swarms by means of parallel computing, for which the term deep swarming (see [Ultsch, 2016b]) is introduced in this work in analogy to deep learning [Goodfellow et al., 2016]. Additionally, using the prabclus package, the largest withincluster gap, the cluster separation, and the average within-cluster dissimilarity of [Hennig, 2014] were calculated to be $0.5,0.33$ and 0.29 , respectively. These values are the minima reported in [Hennig, 2014], presented there in Fig. 4. Seven clusters of the average linkage hierarchical clustering with ten clusters ([Hennig, 2014, p. 5]) could be reproduced (see supplement $\mathrm{H}$ ) with a total accuracy of $93 \%$. Finally, as Figure 11.8 shows, the clusters strongly depend on the geographic origins of the bees:

"Longitude (x-axis) and latitude (y-axis) of locations of individuals in decimal format, i.e. one number is latitude (negative values are South), with minutes and seconds converted to fractions. The other number is longitude (negative values are West)" (see [Hennig, 2014] and the prabclus package).

After the transformation into a two-dimensional plane Figure 11.8 shows that the first eight clusters (96\% of data) are consistent with the geography (top) except for the Outliers in Queensland (bottom). The dependency on geography was also illustrated in [Franck et al., 2004, p. 2319].

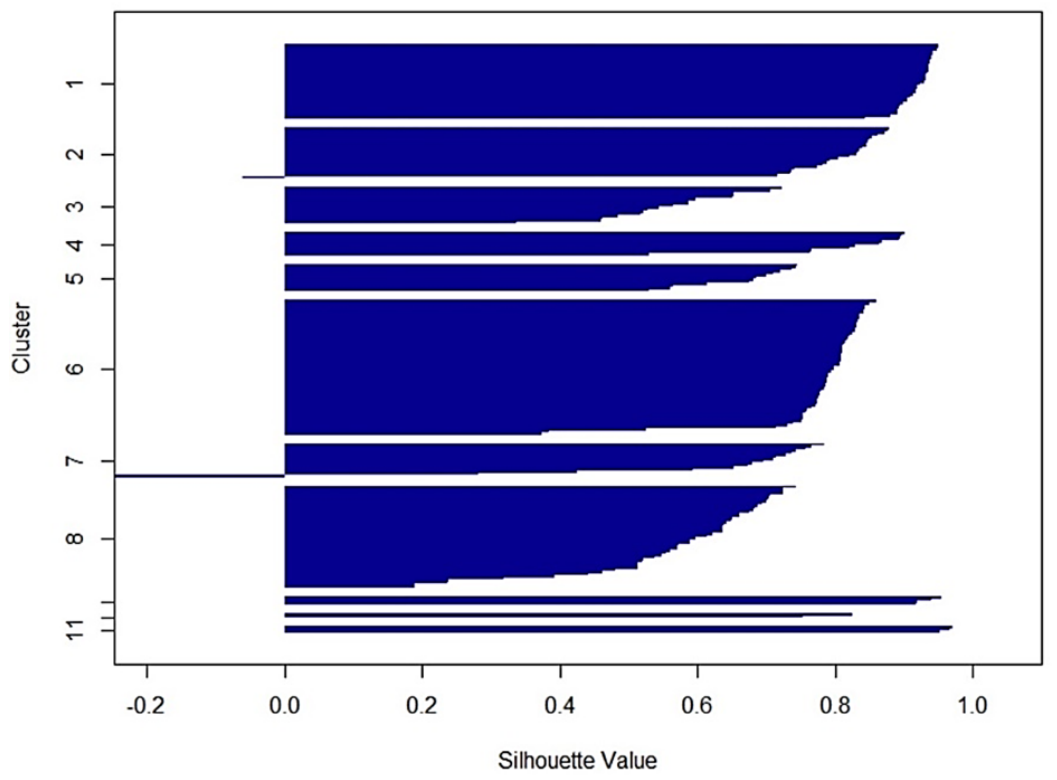

Figure 11.6: Silhouette plot of the Tetragonula data set, showing very homogeneous cluster structures because most of the data points (y-axis) are above a value of 0.5 (x-axis). 


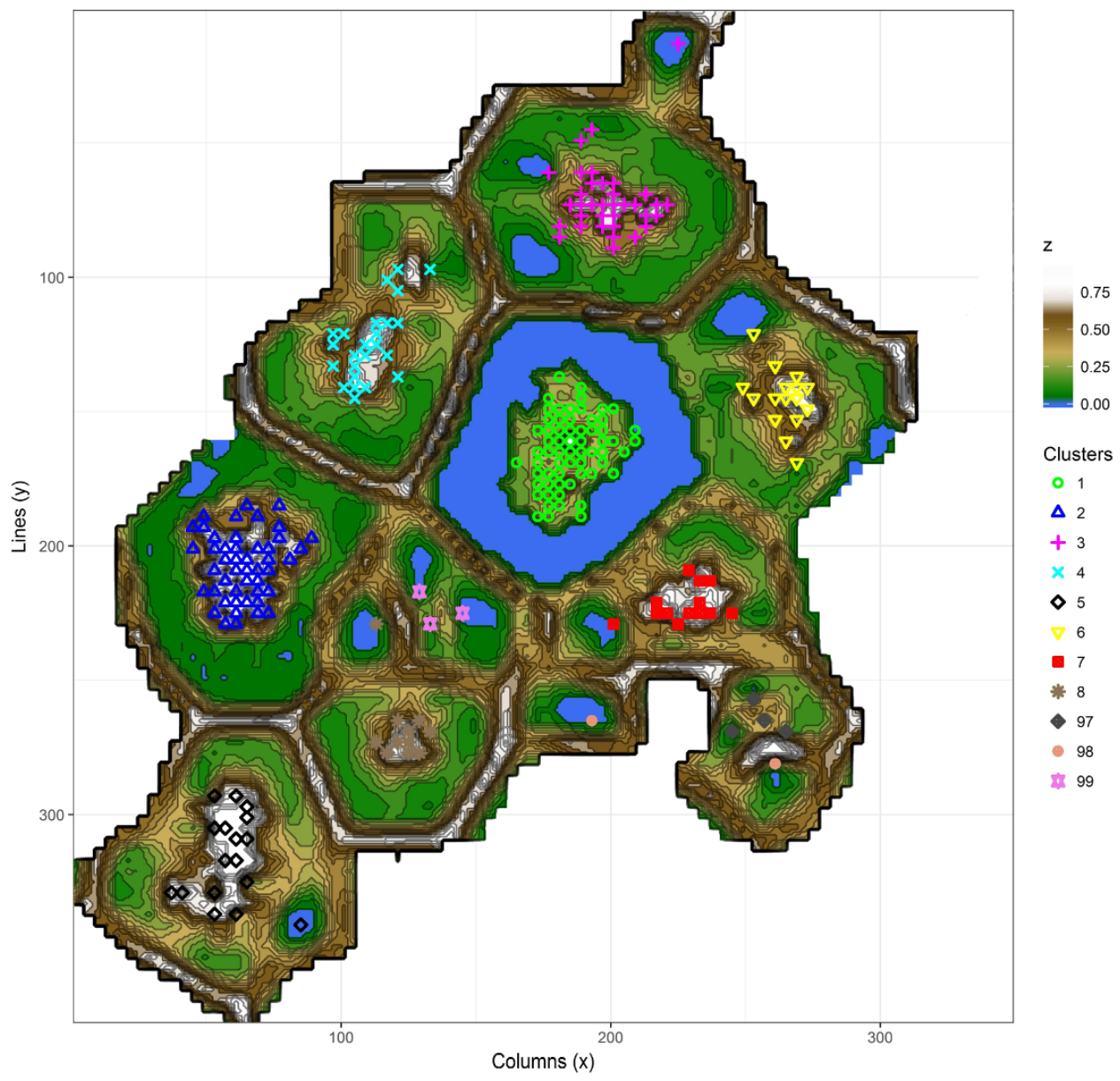

Figure 11.7: Topographic map of the DBS clustering of the Tetragonula data set with the best DCE shows eight clusters and three groups of outliers. The cluster labels are colored as shown on the right, and a similar color code is used in Figure 11.8 below. Clusters are ordered sequentially by the number of samples such that in cluster 1 lies the bee species with the highest occurrence. 

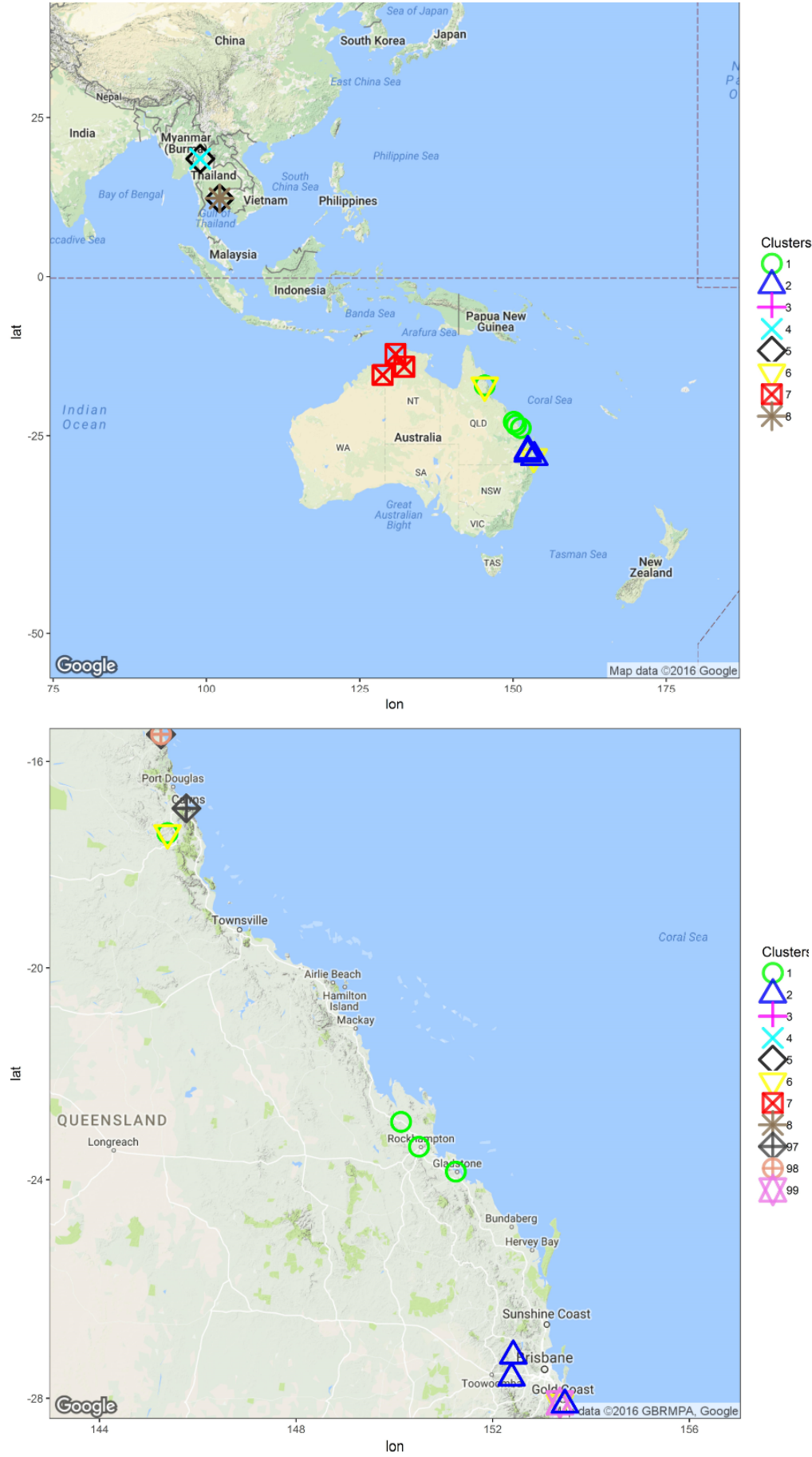

Figure 11.8: Clustering is consistent with the geographic origins: The first eight clusters ( $96 \%$ of data) are consistent with the geography (top) except for the Outliers in Queensland (bottom). Pictures were generated using the ggmap CRAN package. 


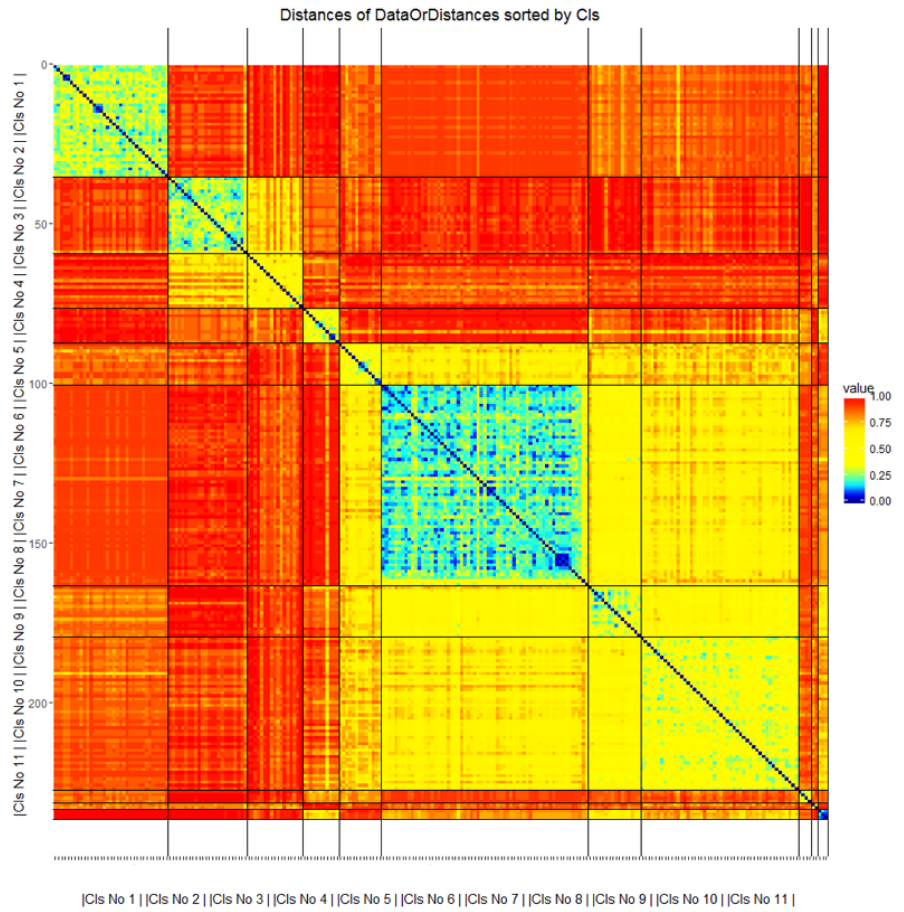

Figure 11.9: Heatmap of the distances for the Tetragonula data set shows large intercluster distances.

Open Access This chapter is licensed under the terms of the Creative Commons Attribution 4.0 International License (http://creativecommons.org/licenses/by/4.0/), which permits use, sharing, adaptation, distribution and reproduction in any medium or format, as long as you give appropriate credit to the original author(s) and the source, provide a link to the Creative Commons license and indicate if changes were made.

The images or other third party material in this chapter are included in the chapter's Creative Commons license, unless indicated otherwise in a credit line to the material. If material is not included in the chapter's Creative Commons license and your intended use is not permitted by statutory regulation or exceeds the permitted use, you will need to obtain permission directly from the copyright holder. 